\title{
Direct and Inverse Problems for Thermal Grooving by Surface Diffusion with Time Dependent Mullins Coefficient
}

\author{
Mansur I. Ismailov ${ }^{a, b}$ \\ ${ }^{a}$ Department of Mathematics, Gebze Technical University \\ 41400 Gebze-Kocaeli, Turkey \\ ${ }^{b}$ Department of Mathematics, Khazar University \\ AZ1096 Baku, Azerbaijan \\ E-mail(corresp.): mismailov@gtu.edu.tr
}

Received April 8, 2020; revised November 29, 2020; accepted November 30, 2020

\begin{abstract}
We consider the Mullins' equation of a single surface grooving when the surface diffusion is not considered as very slow. This problem can be formed by a surface grooving of profiles in a finite space region. The finiteness of the space region allows to apply the Fourier series analysis for one groove and also to consider the Mullins coefficient as well as slope of the groove root to be time-dependent. We also solve the inverse problem of finding time-dependent Mullins coefficient from total mass measurement. For both of these problems, the grooving side boundary conditions are identical to those of Mullins, and the opposite boundary is accompanied by a zero position and zero curvature which both together arrive at self adjoint boundary conditions.
\end{abstract}

Keywords: Mullins' equation, initial-boundary value problem, inverse coefficient problem, Fourier method.

AMS Subject Classification: 35R30; 35K35; 35K05; 42B05.

\section{Introduction and problem formulation}

The paper by Mullins [12] considers the problem of calculation of the time evolution of the free surface in the process when a vertical flat grain boundary meets a horizontal flat surface. The grain boundary forms a groove in the surface, with a known angle and the groove becomes deeper with time.

If we suppose that the surface profile is defined by a function $p(x, t)$, where $x$ is the horizontal coordinate and $t$ is time, Mullins [12] showed that the idealized

Copyright (C) 2021 The Author(s). Published by Vilnius Gediminas Technical University

This is an Open Access article distributed under the terms of the Creative Commons Attribution License (http://creativecommons.org/licenses/by/4.0/), which permits unrestricted use, distribution, and reproduction in any medium, provided the original author and source are credited. 
small-slope approximation case $\left(1+\left(p_{x}\right)^{2}\right)^{-\frac{1}{2}} \simeq 1$ becomes a linear equation for the evolution of the surface profile $p(x, t)$,

$$
p_{t}+B p_{x x x x}=0,
$$

where $B=D_{s} \gamma_{s} \omega /(k \tau)$ is Mullins coefficient, with surface diffusivity $D_{s}$, surface energy $\gamma_{s}$, the atomic volume $\omega$, Boltzmann constant $k$, and the absolute temperature $\tau$. In most crystalline systems, $\gamma_{s}$ is much larger than typical interfacial energies $\gamma_{i}$. It can be considered that the surface diffusion $D_{s}$ taking place in some chemical phenomenon may be depend on time.

The alternative way arriving to time dependent Mullins coefficient model can be taken by more standard non-linear form of Mullins' equation [3]

$$
\theta_{t}=-\partial_{x}^{2}\left(D(\theta) \partial_{x}\left(E(\theta) \theta_{x}\right)\right),
$$

where $\theta=p_{x}$. Here $D$ and $E$ are surface diffusivity and tension coefficients and they may be general depend on the angle between the material surface and the crystal planes for an anisotropic material. For a polycrystalline metal, the surface diffusivity, the solid-fluid interfacial tension and the grain-boundary tension depend on temperature. Therefore, it should be extended to allow for transport coefficients to depend explicitly on time, as well as on slope in classic Mullins' equation. An alternative interpretation of the equation (1.1) with constant coefficient is the case with both $D$ and $E$ are constants and with time dependent coefficient is the case with both $D$ and $E$ are solely time dependent coefficients. It should be mentioned that a non-linear forward problem for the grain boundary with time-dependent Mullins coefficient due to temperature changes, has been solved in $[3,14]$.

Mullins [12] considers (1.1) for a groove, located at $x=0$. It is mentioned in [1] that, if the surface diffusion is considered as very slow then it allows the boundary conditions for (1.1) to be changed from finite spatial range $x=l$ to infinite $x \rightarrow+\infty$. Mullins determined the symmetric semi-infinite groove profile due to a single interface at $x=0$ by solving (1.1) in the quadrant $x>0$, $t>0$, subject to initial and boundray conditions

$$
\begin{aligned}
& \left.p\right|_{t=0}, \quad x>0, \\
& \left.p_{x}\right|_{x=0}=m,\left.\quad p_{x x x}\right|_{x=0}=0, \quad t>0,
\end{aligned}
$$

where $m$ is the slope of the groove root, is the equilibrium dihedral angle at the surface-interface junction given by $m=\frac{\gamma_{i}}{2 \gamma_{s}}$. The condition (1.2) describes the surface evolves from a flat surface. The first condition (1.3) reflects the symmetry of the system when the grain boundary is vertical. The second condition (1.3) imposes the zero flux at the grain boundary.

There are plenty of studies focusing on the solution of linear Mullins' equation (1.1) with constant coefficient. Mullins [12] solved this problem using Laplace transforms with respect to $t$. However, it is much simpler to use a Fourier cosine transform with respect to $x$ [11]. This paper also solved the problem for an infinite periodic row of grooves that develops the Fourier series solution of published in earlier paper [14], where a sinusoidal disturbance was 
considered, leading to a dispersion relation. A multiple integration technique of the integral-balance method also allows to solve this problem in [8]. In the papers $[2,6,10]$ the Mullins' equation is considered in $\mathbb{R} /\{0\}, t>0$ and using various self-similar solution conception they found solution for $\mathbb{R} /\{0\}, t>0$. We can also mention [7,9] for the fractional sub-diffusion modelling of Mullins' equation.

The equation (1.1) and boundary conditions (1.3) are identical to those of Mullins. The boundary conditions can not be always changed from $x=l$ to $x \rightarrow+\infty$ if the surface diffusion is not considered as very slow. In present case the boundary $x=l$ can be supported by a zero position and zero curvature at that boundary:

$$
\left.p\right|_{x=l}=0,\left.\quad p_{x x}\right|_{x=l}=0, \quad t>0 .
$$

The Laplace method is a suitable method for a constant choice of Mullins coefficient $B$ and also groove profile at the root has be a fixed slope $m$ for all time, but generally they have no obligation to be constant with respect to time variable. The finite range consideration of space variable $x$ allows to take Mullins coefficient and slope of the groove root time dependent.

The spatial and time finite range version of Mullins equation with Mullins grooving boundaries (1.3) at $x=0$ and also zero position and zero curvature boundaries (1.4) at $x=l$ can be formulated in following form:

$$
\begin{aligned}
& u_{t}+B u_{x x x x}=f(x, t), \quad(x, t) \in \Omega_{T}, \\
& \left.u_{x}\right|_{x=0}=0,\left.\quad u_{x x x}\right|_{x=0}=0,\left.\quad u\right|_{x=l}=0,\left.\quad u_{x x}\right|_{x=l}=0, t \in[0, T], \\
& \left.u\right|_{t=0}=\phi(x), x \in[0, l]
\end{aligned}
$$

by the substation

$$
p(x, t)=u(x, t)+v(x, t),
$$

where $v(x, t)$ is a function satisfying the conditions $\left.v_{x}\right|_{x=0}=m,\left.v_{x x x}\right|_{x=0}=$ $0,\left.v\right|_{x=l}=0,\left.v_{x x}\right|_{x=l}=0$ and $\Omega_{T} \equiv\{(x, t): 0<x<l ; 0<t \leq T\}$.

The total mass integral condition

$$
\int_{0}^{l} u(x, t) d x=E(t), t \in[0, T]
$$

will be used in inverse problem.

DP: Let $B(t), t \in[0, T]$ be a known positive function. We call direct problem the problem of finding $u(x, t)$ satisfying the equations (1.5)-(1.7).

IP: Let $B(t)$ be unknown function. We call inverse problem the problem of finding the pair $(B(t), u(x, t))$ satisfying the equations (1.5)-(1.7) and (1.8) with $B(t)>0, t \in[0, T]$.

We establish conditions for the existence and uniqueness of a smooth (classical) solutions both direct and inverse problems. The finite range for $x$ suggests the method of separation of variables. For the simplicity we will suppose that $l=1$. 
The auxiliary spectral problem of the IBVP (1.5)-(1.7) is

$$
\left\{\begin{array}{l}
y^{\prime \prime \prime \prime}(x)=\lambda y(x), 0 \leq x \leq 1 \\
y^{\prime}(0)=0, y^{\prime \prime \prime}(0)=0 \\
y(1)=0, y^{\prime \prime}(1)=0
\end{array}\right.
$$

The paper is organized as follows. In Section 2, we recall the eigenvalues and eigen-functions of the auxiliary spectral problem and existence-uniqueness theorem for DP (1.5)-(1.7). In Section 3, the existence and uniqueness theorems of the IP (1.5)-(1.8) are proved.

\section{DP: Fourier series representation of the solution}

Let $B(t) \in C[0, T]$ be a known function with $0<\underline{b} \leq B(t) \leq \bar{b}$, where $\underline{b}$ and $\bar{b}$ are constants. The smoothness conditions $f(x, t) \in C\left(\bar{\Omega}_{T}\right), \varphi(x) \in C^{2}[0,1]$ and the consistency conditions

$$
\varphi(0)=0, \varphi^{\prime}(0)+\alpha \varphi^{\prime \prime}(1)=0
$$

are necessary for the existence of a classical solution $u(x, t) \in C^{4,1}\left(\Omega_{T}\right) \cap$ $C^{3,0}\left(\bar{\Omega}_{T}\right)$ of the problem $(1.5)-(1.7)$.

First, consider the spectral problem (1.9) which is generated by differential operator

$$
L(y) \equiv y^{\prime \prime \prime \prime}, 0<x<1
$$

with the domain

$$
D(L) \equiv\left\{y \in C^{4}[0,1]: y^{\prime}(0)=0, y^{\prime \prime \prime}(0)=0, y(1)=0, y^{\prime \prime}(1)=0\right\} .
$$

For arbitrary $y, z \in D(L)$

$$
(L(y), z) \equiv-\int_{0}^{1} y^{\prime \prime \prime \prime}(x) z(x) d x=-\int_{0}^{1} y(x) z^{\prime \prime \prime \prime}(x) d x \equiv(y, L(z))
$$

holds. It means that operator $L$ is self-adjoint then the eigenvalues are real and normalized eigenfunctions are orthonormal basis in the space $L^{2}[0,1][13]$. Moreover, $\lambda=\frac{\left(y^{\prime \prime}, y^{\prime \prime}\right)}{(y, y)}>0$ for arbitrary eigenvalue $\lambda$ and corresponding eigenfunction $y$ that $L(y)=\lambda y, y \in D(L)$.

Simple calculations yields the positive eigenvalues normed eigenfunctions

$$
\lambda_{n}=\left(\frac{\pi}{2}+\pi n\right)^{4}, \quad y_{n}(x)=\sqrt{2} \cos \left(\frac{\pi}{2}+\pi n\right) x, 0,1,2, \ldots
$$

of operator $L$.

The following lemma is useful for DP.

Lemma 1. For $\varphi(x) \in D(L)$ the inequality

$$
\sum_{n=0}^{\infty} \lambda_{n}^{\frac{3}{4}}\left|\varphi_{n}\right| \leq \frac{1}{\sqrt{2}}\left\|\varphi^{\prime \prime \prime \prime}\right\|_{L_{2}(0,1)}
$$

holds, where $\varphi_{n}=\left(\varphi, y_{n}\right)$. 
Proof. It is clear that

$$
\begin{aligned}
\lambda_{n}^{\frac{3}{4}} \varphi_{n} & =\frac{\lambda_{n}}{\sqrt[4]{\lambda_{n}}}\left(\varphi, y_{n}\right)=\frac{1}{\sqrt[4]{\lambda_{n}}}\left(\varphi, \lambda_{n} y_{n}\right)=\frac{1}{\sqrt[4]{\lambda_{n}}}\left(\varphi, y_{n}^{\prime \prime \prime \prime}\right) \\
& =\frac{1}{\sqrt[4]{\lambda_{n}}}\left(\varphi, L\left(y_{n}\right)\right)=\frac{1}{\sqrt[4]{\lambda_{n}}}\left(L(\varphi), y_{n}\right)=\frac{1}{\sqrt[4]{\lambda_{n}}}\left(\varphi^{\prime \prime \prime \prime}, y_{n}\right)
\end{aligned}
$$

By using Cauchy-Shwartz and Bessel inequalities

$$
\begin{aligned}
\sum_{n=0}^{\infty} \lambda_{n}^{\frac{3}{4}}\left|\varphi_{n}\right| & =\sum_{n=1}^{\infty} \frac{1}{\sqrt[4]{\lambda_{n}}}\left|\left(\varphi^{\prime \prime \prime \prime}, y_{n}\right)\right| \leq\left(\sum_{n=1}^{\infty} \frac{1}{\sqrt{\lambda_{n}}}\right)^{\frac{1}{2}}\left(\sum_{n=1}^{\infty}\left|\left(\varphi^{\prime \prime \prime \prime}, y_{n}\right)\right|^{2}\right)^{\frac{1}{2}} \\
& \leq c\left\|\varphi^{\prime \prime \prime \prime}\right\|_{L_{2}(0,1)}
\end{aligned}
$$

holds with $c=\left(\sum_{n=0}^{\infty} \frac{1}{\sqrt{\lambda_{n}}}\right)^{\frac{1}{2}}=\frac{1}{\sqrt{2}}$.

The main result for DP is presented as follows.

Theorem 1. (Existence and uniqueness of DP) Let the following conditions be satisfied: $\varphi(x) \in D(L), f(x, t) \in C\left(\bar{\Omega}_{T}\right)$ and $f(x, t) \in D(L)$ for $\forall t \in$ $[0, T]$. Then the problem $(1.5)-(1.7)$ has a unique solution $u(x, t) \in C^{4,1}\left(\Omega_{T}\right) \cap$ $\left.C^{3,0}\left(\bar{\Omega}_{T}\right)\right)$ of the form $(2.1)$.

Proof. To construct the formal solution of the problem (1.5)-(1.7) for arbitrary $B(t) \in C[0, T]$, we will use the Fourier series in terms of the eigenfunctions $y_{n}(x)=\sqrt{2} \cos \left(\sqrt[4]{\lambda_{n}} x\right), n=0,1,2, \ldots$ of the auxiliary spectral problem (1.9):

$$
u(x, t)=\sum_{n=0}^{\infty}\left[\varphi_{n} e^{-\lambda_{n} \int_{0}^{t} B(s) d s}+\int_{0}^{t} f_{n}(\tau) e^{-\lambda_{n} \int_{\tau}^{t} B(s) d s} d \tau\right] y_{n}(x) .
$$

To show that this series then satisfies all the conditions of the problem (1.5)(1.7), we must prove that the the $t$-partial derivative and the $x x x x$-fourth order partial derivative of the function defined by (2.1) is continuous, satisfies equation (1.5) in the region $0<x<1, t>0$, and the function (2.1) its $x x$ second and $x x x$-third order partial derivative at the boundary points of the region (for points $t=0, x=0, x=1$ ) must be continuous.

We show that the series arising by termwise differentiation

$$
\begin{aligned}
& u_{t}(x, t) \sim-\sum_{n=0}^{\infty}\left[\lambda_{n} \varphi_{n} B(t) e^{-\lambda_{n} \int_{0}^{t} B(s) d s}-f_{n}(t)\right. \\
& \left.+\int_{0}^{t} f_{n}(\tau) \lambda_{n} B(t) e^{-\lambda_{n} \int_{\tau}^{t} B(s) d s} d \tau\right] y_{n}(x) \\
& u_{x x x x}(x, t) \sim \sum_{n=0}^{\infty}\left[\lambda_{n} \varphi_{n} e^{-\lambda_{n} \int_{0}^{t} B(s) d s}+\int_{0}^{t} f_{n}(\tau) \lambda_{n} e^{-\lambda_{n} \int_{\tau}^{t} B(s) d s} d \tau\right] y_{n}(x) .
\end{aligned}
$$


for $t \geq \varepsilon$ ( $\varepsilon$ is an arbitrary positive number) converge uniformly or the simple convergence of the majorant series within constant factor

$$
\sum_{n=0}^{\infty} \lambda_{n} e^{-\lambda_{n} \underline{b} \varepsilon}, \quad \sum_{n=0}^{\infty} \lambda_{n} e^{-\lambda_{n} \underline{b}(\varepsilon-\tau)} \quad \sum_{n=0}^{\infty}\left|f_{n}(t)\right| .
$$

The convergence of first two majorant series results from D'Alambert criterion and the last is result of Lemma 1. Hence, it follows that the series (2.2) and (2.3) for $t \geq \varepsilon>0$ are uniformly convergent. Further, we conclude from superposition principle that the function defined by the series (2.1) satisfies equation (1.5). Since $t$ is arbitrary it holds for all $t>0$.

The function (2.1) and its $t$-first, $x x$-second and $x x x$-third order partial derivative must be continuous at boundary points. More precicisely, the series (2.1) must be continuous at $t=0$,

$$
\begin{aligned}
u_{x x x}(x, t) \sim & \sqrt{2} \sum_{n=0}^{\infty} \lambda_{n}^{\frac{3}{4}}\left[\varphi_{n} e^{-\lambda_{n} \int_{0}^{t} B(s) d s}+\int_{0}^{t} f_{n}(\tau) \lambda_{n} e^{-\lambda_{n} \int_{\tau}^{t} B(s) d s} d \tau\right] \\
& \times \sin \left(\sqrt[4]{\lambda_{n}} x\right)
\end{aligned}
$$

must be continuous at boundary point $x=0$ and

$$
\begin{aligned}
u_{x x}(x, t) \sim & -\sqrt{2} \sum_{n=0}^{\infty} \lambda_{n}^{\frac{1}{2}}\left[\varphi_{n} e^{-\lambda_{n} \int_{0}^{t} B(s) d s}+\int_{0}^{t} f_{n}(\tau) \lambda_{n} e^{-\lambda_{n} \int_{\tau}^{t} B(s) d s} d \tau\right] \\
& \times \cos \left(\sqrt[4]{\lambda_{n}} x\right)
\end{aligned}
$$

must be continuous at boundary point $x=1$. They are sufficient that the majorant series

$$
\sum_{n=0}^{\infty} \lambda_{n}^{\frac{3}{4}}\left|\varphi_{n}\right|, \quad \sum_{n=0}^{\infty} \lambda_{n}^{\frac{3}{4}}\left|f_{n}(\tau)\right|
$$

are convergent. Under the assumption $\varphi(x) \in D(L)$ and $f(x, t) \in D(L)$ for $\forall t \in[0, T]$ the convergence of these series is a result of Lemma 1 .

We therefore obtain a function $\left.u(x, t) \in C^{4,1}\left(\Omega_{T}\right) \cap C^{3,0}\left(\bar{\Omega}_{T}\right)\right)$ which is a solution of the problem (1.5)-(1.7) given by the Fourier series (2.1), that it is unique from the unique Fourier representations of the functions.

\section{IP: Finding time dependent Mullins coefficient}

Let $B(t), 0 \leq t \leq T$ be unknown function. A solution of inverse problem $(1.5)-(1.8)$ is understood as a pair of functions $(B(t), u(x, t))$ from the class $C[0, T] \times C^{4,1}\left(\Omega_{T}\right) \cap C^{3,0}\left(\bar{\Omega}_{T}\right)$, with $B(t)>0, t \in[0, T]$, such that the equation (1.5) and conditions (1.6)-(1.8) are satisfied. The following condition instead of (1.8)

$$
\int_{0}^{1} u_{t}(x, t) d x=E^{\prime}(t), 0 \leq t \leq T
$$

is more applicable for the solution of IP which is obtained from (1.8) under additional conditions on the data. 
Let us introduce the following class of functions

$$
\stackrel{\circ}{C}_{k}[0,1] \equiv \begin{gathered}
\left\{y \in C^{4 k+1}[0,1]: y^{(4 i+1)}(0)=y^{(4 i+3)}(0)=\right. \\
\left.y^{(4 i)}(1)=y^{(4 i+2)}(1)=y^{(4 i+4)}(1)=0, i=0, \ldots, k-1\right\}
\end{gathered}
$$

for arbitrary $k \in \mathbb{N}$.

Lemma 2. For $\varphi(x) \in \stackrel{\circ}{C}_{k}[0,1]$ the inequality

$$
\sum_{n=1}^{\infty} \lambda_{n}^{k}\left|\varphi_{n}\right| \leq \frac{1}{\sqrt{2}}\left\|\varphi^{(4 k+1)}\right\|_{L_{2}(0,1)}
$$

holds, where $\varphi_{n}=\left(\varphi, y_{n}\right)$.

Proof. It is clear that

$$
\lambda_{n}^{k} \varphi_{n}=\left(\varphi^{(4 k)}, y_{n}\right)=-\frac{1}{\sqrt[4]{\lambda_{n}}}\left(\varphi^{(4 k+1)}, z_{n}\right)
$$

by the integration by parts, where $z_{n}=\sqrt{2} \sin \left(\sqrt[4]{\lambda_{n}} x\right)$.

By using Cauchy-Shwartz and Bessel inequalities

$$
\begin{aligned}
\sum_{n=1}^{\infty} \lambda_{n}^{k}\left|\varphi_{n}\right| & =\sum_{n=1}^{\infty} \frac{1}{\sqrt[4]{\lambda_{n}}}\left|\left(\varphi^{(4 k+1)}, z_{n}\right)\right| \\
& \leq\left(\sum_{n=1}^{\infty} \frac{1}{\sqrt{\lambda_{n}}}\right)^{\frac{1}{2}}\left(\sum_{n=1}^{\infty}\left|\left(\varphi^{(4 k+1)}, z_{n}\right)\right|^{2}\right)^{\frac{1}{2}} \leq c\left\|\varphi^{(4 k+1)}\right\|_{L_{2}(0,1)}
\end{aligned}
$$

holds with $c=\left(\sum_{n=1}^{\infty} \frac{1}{\sqrt{\lambda_{n}}}\right)^{\frac{1}{2}}=\frac{1}{\sqrt{2}}$.

The main result for IP are presented as follows.

Theorem 2. (Existence of IP ) Let the following conditions be satisfied:

- $\varphi(x) \in \stackrel{\circ}{C}_{2}[0,1]$ with $(-1)^{n} \varphi_{n}>0$;

- $E(t) \in C^{1}[0,1]$ with $E(t)>0 ; E^{\prime}(t)<0$ and $E(0)=\int_{0}^{1} \varphi(x) d x$;

- $f(x, t) \in C\left(\bar{D}_{T}\right)$ and $f(x, t) \in C_{2}[0,1]$ with $(-1)^{n} f_{n}(t) \geq 0$ for $\forall t \in$ $[0, T]$.

Then the problem (1.5)-(1.8) has a classical solution $(B(t), u(x, t))$ with $u(x, t) \in C^{4,1}\left(\bar{\Omega}_{T}\right)$.

Proof. The series (2.1) can be termwise continuously differentiable by $t$ that the series (2.2) is uniformly converges, since the series $\sum_{n=0}^{\infty} \lambda_{n}\left|\varphi_{n}\right|$, $\sum_{n=0}^{\infty} \lambda_{n}\left|f_{n}(\tau)\right|$ converge by Lemma 2 . Thus the total mass condition (1.8) implies (3.1). The formulas (2.1) and (3.1) yield an equation with respect to

$$
\begin{gathered}
B(t):-\sqrt{2} \sum_{n=0}^{\infty} \frac{(-1)^{n}}{\sqrt[4]{\lambda_{n}}} B(t)\left[\varphi_{n} \lambda_{n} e^{-\lambda_{n} \int_{0}^{t} B(s) d s}+\int_{0}^{t} f_{n}(\tau) \lambda_{n} e^{-\lambda_{n} \int_{\tau}^{t} B(s) d s} d \tau\right] \\
=E^{\prime}(t)-\sqrt{2} \sum_{n=0}^{\infty} \frac{(-1)^{n}}{\sqrt[4]{\lambda_{n}}} f_{n}(t)
\end{gathered}
$$


or

$$
B=\Phi(B)
$$

with $\Phi(B(t))=\frac{-E^{\prime}(t)+\sqrt{2} \sum_{n=0}^{\infty}(-1)^{n} f_{n}(t) / \sqrt[4]{\lambda_{n}}}{\sqrt{2} \sum_{n=0}^{\infty} \frac{(-1)^{n}}{\sqrt[4]{\lambda_{n}}}\left[\varphi_{n} \lambda_{n} e^{-\lambda_{n} \int_{0}^{t} B(s) d s}+\int_{0}^{t} f_{n}(\tau) \lambda_{n} e^{-\lambda_{n} \int_{\tau}^{t} B(s) d s} d \tau\right]}$ since

$$
\int_{0}^{1} y_{n}(x) d x=\frac{\sqrt{2}}{\sqrt[4]{\lambda_{n}}}(-1)^{n}
$$

It is easy to show that $\sqrt{2} \sum_{n=0}^{\infty} \frac{(-1)^{n}}{\sqrt[4]{\lambda_{n}}} f_{n}(t)=\int_{0}^{1} f(x, t) d x$. Let us denote

$$
\underline{b}=\frac{\min _{t \in[0, T]} \int_{0}^{1} f(x, t) d x-\max _{t \in[0, T]} E^{\prime}(t)}{\sqrt{2} \sum_{n=0}^{\infty} \frac{\lambda_{n}}{\sqrt[4]{\lambda_{n}}}\left[\varphi_{n}+\int_{0}^{T} f_{n}(\tau) d \tau\right]}, \bar{b}=\frac{\max _{t \in[0, T]} \int_{0}^{1} f(x, t) d x-\min _{t \in[0, T]} E^{\prime}(t)}{\min _{t \in[0, T]} E(t)} .
$$

Let us introduce the following class of functions:

$$
M \equiv\{B(t) \in C[0, T]: \underline{b} \leq B(t) \leq \bar{b}\} .
$$

It is clear that $\Phi$ maps the set $M$ onto itself, i.e., $\Phi: M \rightarrow M$. Now, we will show that the operator $\Phi$ is uniformly bounded and equicontinuous. Let $M_{1}$ be any bounded subset of the set M. Since $\Phi\left(M_{1}\right) \subset M$, it follows that $\Phi\left(M_{1}\right)$ is uniformly bounded. According to the Arzela theorem, we establish the equicontinuity of the set $\Phi$. For this purpose, we take an arbitrary $\varepsilon>0$ and establish the existence of $\delta>0$ such that

$$
\left|\Phi\left(B\left(t_{1}\right)\right)-\Phi\left(B\left(t_{2}\right)\right)\right|<\varepsilon \text { for }\left|t_{1}-t_{2}\right|<\delta .
$$

Taking into account that

$$
\left|\Phi\left(B\left(t_{1}\right)\right)-\Phi\left(B\left(t_{2}\right)\right)\right| \leq \frac{\left|K\left(t_{1}\right)-K\left(t_{2}\right)\right|}{N\left(t_{2}\right)}+\frac{\left|K\left(t_{1}\right)\left(N\left(t_{1}\right)-N\left(t_{2}\right)\right)\right|}{N\left(t_{1}\right) N\left(t_{2}\right)},
$$

where $K(t)=-E^{\prime}(t)+\int_{0}^{1} f(x, t) d x$ and

$$
N(t)=\sqrt{2} \sum_{n=0}^{\infty} \frac{(-1)^{n}}{\sqrt[4]{\lambda_{n}}}\left[\varphi_{n} \lambda_{n} e^{-\lambda_{n} \int_{0}^{t} B(s) d s}+\int_{0}^{t} f_{n}(\tau) \lambda_{n} e^{-\lambda_{n} \int_{\tau}^{t} B(s) d s} d \tau\right] .
$$

By using the inequality

$$
\left|e^{-\lambda_{n} \int_{\tau}^{t_{1}} B(s) d s}-e^{-\lambda_{n} \int_{\tau}^{t_{2}} B(s) d s}\right| \leq \lambda_{n}\left|t_{1}-t_{2}\right| \max _{t \in[0, T]} B(t)
$$

we get

$$
\left|N\left(t_{1}\right)-N\left(t_{2}\right)\right| \leq \alpha\left|t_{1}-t_{2}\right|
$$

where

$$
\alpha=\sqrt{2}\left(\bar{b} \sum_{n=0}^{\infty} \frac{\lambda_{n}^{2}}{\sqrt[4]{\lambda_{n}}}\left(\left|\varphi_{n}\right|+\int_{0}^{T}\left|f_{n}(\tau)\right| d \tau\right)+\sum_{n=0}^{\infty} \frac{\lambda_{n}}{\sqrt[4]{\lambda_{n}}} \max _{t \in[0, T]}\left|f_{n}(t)\right|\right) .
$$


On the other hand, since $K(t)$ is continuous in the closed interval $[0, T]$, for all $\varepsilon>0$ there exists $\delta_{1}=\delta_{1}(\varepsilon)>0$ such that

$$
\left|K\left(t_{1}\right)-K\left(t_{2}\right)\right| \leq \frac{\varepsilon}{2} \min _{t \in[0, T]} E(t)
$$

for all $t_{1}$ and $t_{2}$ in $[0, T]$ for which $\left|t_{1}-t_{2}\right|<\delta_{1}$. By choosing

$$
\delta=\min \left\{\delta_{1}(\varepsilon), \frac{\left(\min _{t \in[0, T]} E(t)\right)^{2}}{\alpha\left(\max _{t \in[0, T]} \int_{0}^{1} f(x, t) d x-\min _{t \in[0, T]} E^{\prime}(t)\right)} \frac{\varepsilon}{2}\right\}
$$

from (3.3) we obtain that

$$
\left|\Phi\left(B\left(t_{1}\right)\right)-\Phi\left(B\left(t_{2}\right)\right)\right|<\varepsilon
$$

for all $t_{1}$ and $t_{2}$ in $[0, T]$ for which $\left|t_{1}-t_{2}\right|<\delta$. We show that the operator $\Phi$ is uniformly bounded and equicontinuous. Using the Schauder's Fixed Point Theorem, we have a solution $B(t) \in C[0, T]$ of the equation (3.2). Thus, the conditions of the Schauder theorem for mapping $\Phi$ are satisfied, and, hence, there exists a solution $B=B(t)$ of (3.2) from the class $C[0, T]$. Substituting it in $(2.1)$, we find a function $u=u(x, t)$ that, as follows from the proof of the theorem, possesses the required smoothness.

Theorem 3. (Uniqueness of IP) Let the following conditions be satisfied:

- $\varphi(x) \in \stackrel{\circ}{C}_{1}[0,1] ; \quad, E(t) \in C^{1}[0,1]$ with $E(0)=\int_{0}^{1} \varphi(x) d x$

- $f(x, t) \in C\left(\bar{D}_{T}\right)$ and $f(x, t) \in \stackrel{\circ}{C}^{1}[0,1]$ with $\int_{0}^{1} f(x, t) d x \neq E^{\prime}(t)$

for $\forall t \in[0, T]$. Then the solution of the inverse problem (1.5)-(1.8) is unique.

Proof. Assume that there exist two solutions $\left(B_{i}(t), u^{(i)}(x, t)\right), i=1,2$, of problem (1.5)-(1.8). For the difference of these solutions $B(t) \equiv B_{2}(t)-B_{1}(t)$, $u(x, t) \equiv u^{(1)}(x, t)-u^{(2)}(x, t)$, we obtain the following problem:

$$
\begin{aligned}
& u_{t}+B_{1}(t) u_{x x x x}=B(t) u_{x x x x}^{(2)}, 0<x<1 ; 0<t \leq T \\
& \left.u_{x}\right|_{x=0}=0,\left.u_{x x x}\right|_{x=0}=0,\left.u\right|_{x=1}=0,\left.u_{x x}\right|_{x=1}=0,0 \leq t \leq T, \\
& \left.u\right|_{t=0}=0,0 \leq x \leq 1
\end{aligned}
$$

By applying $\int_{0}^{1} u(x, t) d x=0,0 \leq t \leq T$ and using boundary conditions in (3.4) we become to the equality:

$$
B_{1}(t) u_{x x x}(1, t)=B(t) \int_{0}^{1} u_{x x x x}^{(2)}(x, t) d x .
$$

Using the Fourier series representation of $u_{x x x}(1, t)$, we represent a solution of problem (3.4) in the form

$$
-B_{1}(t) 2 \sqrt{2} \sum_{n=0}^{\infty}(-1)^{n} \lambda_{n}^{\frac{3}{4}} \int_{0}^{t} B(t)\left(u_{x x x x}^{(2)}\right)_{n} e^{-\lambda_{n} \int_{\tau}^{t} B_{1}(s) d s} d \tau=B(t) u_{x x x}^{(2)}(1, t) .
$$


Representing $B(t)$ in the form of Volterra integral equation

$$
B(t)+\int_{0}^{t} K(t, \tau) B(\tau) d \tau=0
$$

where

$$
K(t, \tau)=\frac{B_{1}(t) 2 \sqrt{2} \sum_{n=0}^{\infty}(-1)^{n} \lambda_{n}^{\frac{3}{4}}\left(u_{x x x x}^{(2)}\right)_{n} e^{-\lambda_{n} \int_{\tau}^{t} B_{1}(s) d s}}{u_{x x x}^{(2)}(1, t)}
$$

by taking account that $u_{x x x}^{(2)}(1, t) \neq 0$.

From the problem by $u^{(2)}(x, t)$ we get the equality

$$
u_{x x x}^{(2)}(1, t)=\frac{-E_{2}^{\prime}(t)+\int_{0}^{1} f(x, t) d x}{B_{2}(t)}
$$

by applying $\int_{0}^{1} u^{(2)}(x, t) d x=E_{2}(t)$.

The kernel $K(t, \tau)$ is continuous. In view of the properties of Volterra integral equations of the second kind, (3.5) has only the trivial solution $B(t) \equiv$ 0 . Therefore, $B(t) \equiv 0, t \in[0, T]$, and $u(x, t) \equiv 0,(x, t) \in \Omega_{T}$, as a solution of the problem (3.4).

Remark 1. In the case of two grooves located at the points $x=0$ and $x=1$ the both boundaries are supported by the Mullins' conditions which is proposed in Martin [11], namely

$$
\left.u_{x}\right|_{x=0}=0,\left.\quad u_{x x x}\right|_{x=0}=0,\left.\quad u\right|_{x=1}=0,\left.\quad u_{x x x}\right|_{x=1}=0 .
$$

This problem has the spectral problem

$$
\left\{\begin{array}{c}
y^{\prime \prime \prime \prime}(x)=\lambda y(x), 0 \leq x \leq 1 \\
y^{\prime}(0)=0, y^{\prime \prime \prime}(0)=0 \\
y^{\prime}(1)=0, y^{\prime \prime \prime}(1)=0
\end{array}\right.
$$

that is also self-adjoint with eigenvalues and normalized eigenfunctions

$$
\lambda_{n}=(\pi n)^{4} ; y_{n}(x)=\sqrt{2} \cos (\pi n x), n=0,1,2, \ldots
$$

The inverse problem of finding Mullins coefficient has a solution iff consistency-type condition

$$
E^{\prime}(t)=\int_{0}^{1} f(x, t) d x
$$

is satisfied. If the inverse problem has solution then it is not unique. It means that Mullins coefficient can not be uniquely controlled by the total mass in the case of two grooves.

The unique restoration of the time-dependent Mullins coefficient can be obtained by the the measurement of the profile

$$
u\left(x_{0}, t\right)=E(t), t \in[0, T]
$$

at a fixed point $x_{0} \in[0,1]$, in [4]. 
Remark 2. The grooving boundary conditions proposed in Amram et al. [1], namely $\left.\quad u_{x}\right|_{x=0}=0,\left.\quad u_{x x}\right|_{x=0}=0$ as zero flux and zero curvature at the root. The relevant spectral problem can not be self-adjoint for any given boundary condition at $x=1$. By the way the classical theory [5] of expansion in terms of eigenfunctions can not be applicable. The Sturm-type boundary conditions at $x=1$ as $\left.\frac{\partial^{m_{1}}}{\partial x^{m_{1}}} u\right|_{x=1}+\left.\sum_{i=0}^{m_{1}-1} a_{i} \frac{\partial^{i}}{\partial x i} u\right|_{x=1}=0$, $\left.\frac{\partial^{m_{2}}}{\partial x^{m_{2}}} u\right|_{x=1}+\left.\sum_{i=0}^{m_{2}-1} b_{i} \frac{\partial^{i}}{\partial x^{i}} u\right|_{x=1}=0$, where $3 \geq m_{1}>m_{2} \geq 0$ is strongly regular, [13], together with the Amram conditions at $x=0$ that the system of eigenfunctions and associated functions is Riesz basis in $L_{2}[0,1]$.

The Fourier method approach to this problem needs expansion of given functions in terms of eigenfunctions and associated functions of relevant spectral problem.

\section{Conclusions}

We investigate the Mullins' equation of single surface grooving [12] when surface diffusion is not considered as very slow. This problem can be formed by a surface grooving of profiles in finite space region, [1]. The finiteness of the space region allows to apply the Fourier series analysis of finding profile and also to consider the Mullins coefficient time-dependent. We solve the inverse problem of finding time-dependent Mullins coefficient from the total mass data. The grooving boundary are supported by the conditions that are identical to those of Mullins [12] and the opposite boundary supported by the boundary conditions (1.4) which the auxiliary spectral problem is self-adjoint.

In practice, surface diffusivity $D_{s}$ is measured using sophisticated methods of characterisation based on radiotracers, field ion microscopy or topographic techniques. However, if an additional chemical phenomenon is taking place then, the surface diffusion $D s$ depends on time and becomes unknown. In such a situation, the measurement of surface diffusivity depending on time becomes unfeasible using the current state-of-the-art experimental procedures, but instead, one can consider the computational mathematics inversion for its determination. We also mention that, a more fulsome account of the inverse problem would use the small $-t$ part of such a solution, perhaps with random errors added on, as a bench test.

Mullins' condition that there is no diffusion into the grain boundary (zero flux $\left.u_{x x x}\right|_{x=0}=0$ at the grooving root), is replaced in [1] by a zero curvature at the root $\left(\left.u_{x x}\right|_{x=0}=0\right)$. Because the relevant spectral problem can not be selfadjoint for any given boundary condition at $x=1$, the Sturm-type boundary condition at $x=1$ can be accompanied and the Mullins coefficient can also be determined in this case, which suggests a line for further investigation.

\section{References}

[1] D. Amram, L. Klinger, N. Gazit, H. Gluska and E. Rabkin. Grain boundary grooving in thin films revisited: The role of interface diffusion. Acta Materialia, 69:386-396, 2014. https://doi.org/10.1016/j.actamat.2014.02.008. 
[2] T. Asai and Y. Giga. On self-similar solutions to the surface diffusion flow equations with contact angle boundary conditions. Hokkaido University Preprint Series in Mathematics, 1039:1-25, 2013.

[3] P. Broadbridge and J.M. Goard. Temperature-dependent surface diffusion near a grain boundary. Journal of Engineering Mathematics, 66(1-3):87-102, 2010. https://doi.org/10.1007/s10665-009-9332-9.

[4] K. Cao, D. Lesnic and M.I. Ismailov. Determination of the time-dependent thermal grooving coefficient. Journal of Applied Mathematics and Computing, pp. 1-23, 2020. https://doi.org/10.1007/s12190-020-01388-7.

[5] E.A. Coddington and N. Levinson. Theory of Ordinary Differential Equations. McGraw Hill Book Co., Inc., New York, Toronto, London, 1955.

[6] N. Hamamuki. Asymptotically self-similar solutions to curvature flow equations with prescribed contact angle and their applications to groove profiles due to evaporation-condensation. Advances in Differential Equations, 19(3-4):317-358, 2014 .

[7] M. Abu Hamed and A.A. Nepomnyashchy. Groove growth by surface subdiffusion. Physica D: Nonlinear Phenomena, 298-299:42-47, 2015. https://doi.org/10.1016/j.physd.2015.02.001.

[8] J. Hristov. Multiple integral-balance method: Basic idea and an example with Mullins model of thermal grooving. Thermal science, 21(3):1555-1560, 2017. https://doi.org/10.2298/TSCI170410124H.

[9] J. Hristov. Fourth-order fractional diffusion model of thermal grooving: integral approach to approximate closed form solution of the Mullins mode. Mathematical Modelling of Natural Phenomen, 13(1):14, 2018. https://doi.org/10.1051/mmnp/2017080.

[10] H. Kalantarova and A. Novick-Cohen. Self-similar grooving solutions to the Mullins' equation. Quarterly of applied Mathematics, 2019. https://doi.org/10.1090/qam/1570.

[11] P. Martin. Thermal grooving by surface diffusion: Mullins revisited and extended to multiple grooves. Quarterly of applied mathematics, 67(1):125-136, 2009. https://doi.org/10.1090/S0033-569X-09-01086-4.

[12] W.W. Mullins. Theory of thermal grooving. Journal of Applied Physics, 28(3):333-339, 1957. https://doi.org/10.1063/1.1722742.

[13] M. Naimark. Linear Differential Operators. Elementary theory of linear differential operators. New York: Frederick Ungar Publishing Co, 1967.

[14] P. Tritscher and P. Broadbridge. Grain boundary grooving by surface diffusion: an analytic nonlinear model for a symmetric groove. Proceedings of the Royal Society of London. Series A: Mathematical and Physical Sciences, 450(1940):569587, 1995. https://doi.org/10.1098/rspa.1995.0101. 